\title{
Observer Design for Multiphase Flow in Vertical Pipes with Gas-Lift - Theory and Experiments *
}

\author{
O.M. Aamo*, G.O. Eikrem, H.B. Siahaan, B.A. Foss \\ Department of Engineering Cybernetics, Norwegian University of Science and \\ Technology, 7491 Trondheim, Norway
}

\begin{abstract}
Unstable regimes occuring for multiphase flow in vertical risers have successfully been stabilized using conventional linear control techniques. However, these control systems rely on downhole measurements which are at best unreliable, if at all available. In this paper, we design a nonlinear observer for the states of the multiphase flow that relies on topside measurements only, and apply it to estimate downhole pressure for feedback control. A key feature of the design is that it exploits the structure of the model to obtain robustness with respect to the internal flows in the system. Combining the nonlinear observer with conventional PI control of the downhole pressure, we demonstrate in laboratory experiments the potential for increasing production from gas-lift wells by stabilizing the multiphase flow.
\end{abstract}

Key words: Nonlinear observer design, Multiphase flow, Petroleum technology

\section{Introduction}

Pipelines and oil wells with highly oscillatory flow constitute a significant problem in the petroleum industry, and efforts to find inexpensive solutions based on automatic control have increased both in academia and industry $[8,5,12,6,15,13,11]$. Several different instability phenomena related to oil and gas pipelines and wells exist. This study will investigate one such phenomenon: unstable gas-lift wells. Gas-lift is a technology that reduces the hydrostatic

\footnotetext{
* This work was supported by the Gas Technology Center and Petronics at NTNU, and the Norwegian Research Council.

* Corresponding author. E-mail: aamo@itk.ntnu.no.
} 
pressure in the tubing, facilitating production from wells with low reservoir pressure. Gas is injected into the tubing, as deep as possible, and mixes with the fluid from the reservoir, see Figure 1. Since the gas has lower density than

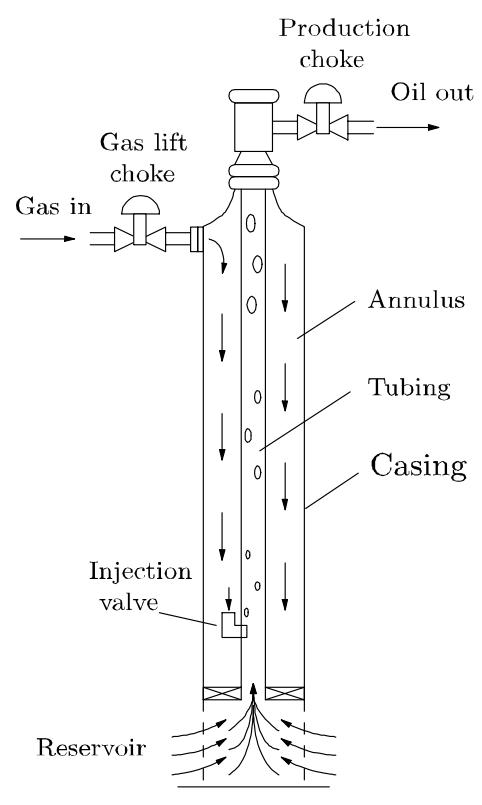

Fig. 1. A gas-lift oil well

the reservoir fluid, the density of the fluid in the tubing, and consequently the downhole pressure (DHP), decrease. When the downhole pressure decreases, the production from the reservoir increases. The lift gas is routed from the surface and into the annulus, which is the volume between the casing and the tubing, and enters the tubing through a valve, or an injection orifice. Backflow from the tubing into the annulus is not permitted by this valve. The dynamics of highly oscillatory flow in gas-lift wells can be described as follows:

(1) Gas from the annulus starts to flow into the tubing. As gas enters the tubing the pressure in the tubing falls, accelerating the inflow of lift-gas.

(2) The gas pushes the major part of the liquid out of the tubing, while the pressure in the annulus falls dramatically.

(3) The annulus is practically empty, and the gas flow into the tubing is blocked by liquid accumulating in the tubing. Due to the blockage, the tubing becomes filled with liquid and the annulus with gas.

(4) Eventually, the pressure in the annulus becomes high enough for gas to penetrate into the tubing, and a new cycle starts.

For more information on this type of instability, often termed casing-heading instability, leading to severe slugging, see [16].

There are in principle two approaches to eliminate highly oscillating flow in gas-lift wells. The first approach is to increase the pressure drop caused by friction. Here it is possible to increase the gas flow rate, reduce the opening 


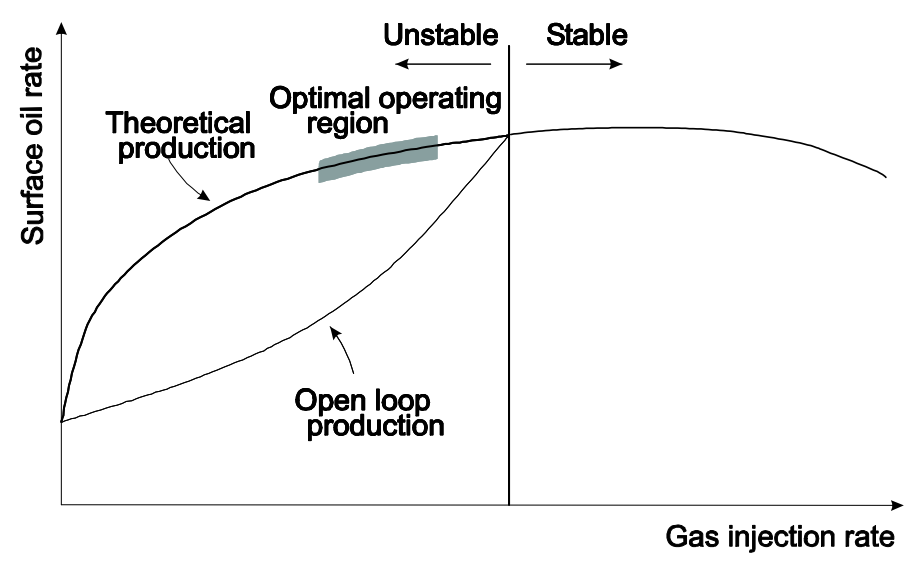

Fig. 2. Gas-lift curve with the region of optimum lift gas utilization.

of the production choke, reduce the size of the gas-lift valve, or decouple the dynamics of the annulus and tubing by obtaining supercritical flow through the injection valve. The second approach is the use of active control to stabilize the well flow, which is the subject of this study. Figure 2 shows a conceptual gas-lift production curve. The produced oil rate is a function of the flow rate of gas injected into the well. The curve shows conditions under which the well exhibits stable or highly oscillatory flow. It is important to note that the average production rate may be significantly lower with unstable well flow, than with stable well flow. This is illustrated by contrasting the "open loop production" to the "theoretical production" curves in Figure 2. The region of optimum lift gas utilization may lie in the unstable region. In addition to causing lower oil production, large oscillations in the flow rate from the well complicate downstream gas/oil/water separation, and may even cause flaring.

Stabilization of gas-lift wells using conventional control techniques has been studied for single well systems in $[1,9,10]$, and for a two-well system in [3]. In [7], a state feedback control law was designed using Lyapunov theory, and the controller was used in an output feedback setting with an extended Kalman filter in [4]. In this paper, we design a nonlinear observer for the states of the multiphase flow in the tubing, and apply it to estimate downhole pressure for feedback control. The design exploits the structure of the model to obtain robustness with respect to the internal flow between the annulus and the tubing.

The paper is organized as follows: In Section 2 we present a mathematical model of the gas-lift well due to [4,7]; in Section 3 we design the observer and apply it in open-loop simulations; in Section 4 an output feedback stabilization scheme combining the nonlinear observer with PI control of the estimated downhole pressure is proposed, and; Section 5 presents experimental results using this controller. Concluding remarks are offered in Section 6 . 


\section{Mathematical Model}

The process described in the introduction, and sketched in Figure 1, is modelled mathematically by three states: $x_{1}$ is the mass of gas in the annulus; $x_{2}$ is the mass of gas in the tubing, and; $x_{3}$ is the mass of oil in the tubing. Looking at Figure 1, we have

$$
\begin{aligned}
& \dot{x}_{1}=w_{g c}-w_{i v}, \\
& \dot{x}_{2}=w_{i v}-w_{p g}, \\
& \dot{x}_{3}=w_{r}-w_{p o},
\end{aligned}
$$

where $w_{g c}$ is a constant mass flow rate of lift gas into the annulus, $w_{i v}$ is the mass flow rate of lift gas from the annulus into the tubing, $w_{p g}$ is the mass flow rate of gas through the production choke, $w_{r}$ is the oil mass flow rate from the reservoir into the tubing, and $w_{p o}$ is the mass flow rate of produced oil through the production choke. The flows are modeled by

$$
\begin{aligned}
w_{g c} & =\text { constant flow rate of lift gas, } \\
w_{i v} & =C_{i v} \sqrt{\rho_{a, i} \max \left\{0, p_{a, i}-p_{t, i}\right\}}, \\
w_{p c} & =C_{p c} \sqrt{\rho_{m} \max \left\{0, p_{t}-p_{s}\right\}} u \\
w_{p g} & =\frac{x_{2}}{x_{2}+x_{3}} w_{p c} \\
w_{p o} & =\frac{x_{3}}{x_{2}+x_{3}} w_{p c} \\
w_{r} & =C_{r}\left(p_{r}-p_{t, b}\right)
\end{aligned}
$$

$C_{i v}, C_{p c}$, and $C_{r}$ are constants, $u$ is the production choke opening $(u(t) \in$ $[0,1]), \rho_{a, i}$ is the density of gas in the annulus at the injection point, $\rho_{m}$ is the density of the oil/gas mixture at the top of the tubing, $p_{a, i}$ is the pressure in the annulus at the injection point, $p_{t, i}$ is the pressure in the tubing at the gas injection point, $p_{t}$ is the pressure at the top of the tubing, $p_{s}$ is the pressure in the separator, $p_{r}$ is the pressure in the reservoir, and $p_{t, b}$ is the pressure at the bottom of the tubing. The separator pressure, $p_{s}$, is assumed to be held constant by a control system, and the reservoir pressure, $p_{r}$, is assumed to be slowly varying and therefore treated as constant. Note that flow rates through the valves are restricted to be positive. The densities are modelled as follows

$$
\begin{aligned}
\rho_{a, i} & =\frac{M}{R T_{a}} p_{a, i}, \\
\rho_{m} & =\frac{x_{2}+x_{3}-\rho_{o} L_{r} A_{r}}{L_{t} A_{t}},
\end{aligned}
$$


and the pressures as follows

$$
\begin{aligned}
p_{a, i} & =\left(\frac{R T_{a}}{V_{a} M}+\frac{g L_{a}}{V_{a}}\right) x_{1}, \\
p_{t} & =\frac{R T_{t}}{M} \frac{x_{2}}{L_{t} A_{t}+L_{r} A_{r}-\nu_{o} x_{3}}, \\
p_{t, i} & =p_{t}+\frac{g}{A_{t}}\left(x_{2}+x_{3}-\rho_{o} L_{r} A_{r}\right), \\
p_{t, b} & =p_{t, i}+\rho_{o} g L_{r} .
\end{aligned}
$$

$M$ is the molar weight of the gas, $R$ is the gas constant, $T_{a}$ is the temperature in the annulus, $T_{t}$ is the temperature in the tubing, $V_{a}$ is the volume of the annulus, $V_{t}$ is the volume of the tubing, $L_{a}$ is the length of the annulus, $L_{t}$ is the length of the tubing, $A_{t}$ is the cross sectional area of the tubing above the injection point, $L_{r}$ is the length from the reservoir to the gas injection point, $A_{r}$ is the cross sectional area of the tubing below the injection point, $g$ is the gravity constant, $\rho_{o}$ is the density of the oil, and $\nu_{o}$ is the specific volume of the oil. The molar weight of the gas, $M$, the density of oil, $\rho_{o}$, and the temperatures, $T_{a}$ and $T_{t}$ are assumed slowly varying and therefore treated as constants.

In summary, the model covers the following case:

- Two-phase flow in the tubing, treating oil and water as a single phase;

- No flashing effects;

- Low gas-to-oil ratio (GOR), reflected in the fact that the flow from the reservoir is modelled as pure oil, and;

- Slowly varying components of gas and oil.

The dynamics of the model has been compared to that of the OLGA $2000^{1}$ multiphase flow simulator in [7], and found to be in satisfactory agreement. It should be noted, however, that the simplicity of the model is a result of the modelling objective, which is to adequately capture the casing-heading instability. A number of other instabilities may occur in gas-lifted oil wells, for instance tubing-heading instability, tubing-reservoir interactions, and hydrodynamic slugging, but these are not captured by this model.

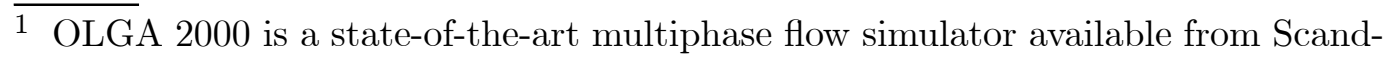
power AS.

http://www.olga2000.com 


\section{State Estimation}

In practice, measurements downhole in the tubing or annulus will in general not be available. If they are available, they must be considered unreliable due to the harsh conditions in which the sensors operate, and the fact that maintainance of the sensors is virtually impossible (a very high failure rate of installed pressure sensors is reported by Statoil [14]). Thus, we will in this work assume that we have measurements at the top of the annulus and tubing, only. The main challenge is how to deal with the multiphase flow in the tubing, whereas the single phase flow in the annulus can accurately be estimated based on one pressure measurement and one temperature measurement. Thus, we will assume that $x_{1}$ is measured. For estimation of the two remaining states, we measure the pressure at the top of the tubing, and either the flow through the production choke or the density at the top of the tubing. Our measurements are therefore

$$
y_{1}(t)=x_{1}(t), y_{2}(t)=p_{t}(t) \text {, and } y_{3}(t)=w_{p c}(t) \text { or } y_{3}(t)=\rho_{m}(t) \text {. }
$$

\subsection{Reduced Order Observer Design}

Since the mass of gas in the annulus can be considered a measurement, we design a reduced order observer for the remaining two states. Before we state our main result, we state key assumptions and intermediate results needed in the convergence proof for the observer.

Assumption 1 The production choke is not allowed to close completely. That $i s$,

$$
u \geq \delta_{u}>0, \forall t \geq 0
$$

Assumption 2 The states are bounded away from zero, and the part of the tubing below the gas injection point is filled with oil. More precisely,

$$
x_{1} \geq \delta_{1}>0, x_{2} \geq \delta_{2}>0, \text { and } x_{3} \geq \rho_{o} L_{r} A_{r}+\delta_{3}>\rho_{o} L_{r} A_{r}, \forall t \geq t_{0} .
$$

Assumption 3 The gas in the tubing has lower density than the oil. More precisely,

$$
L_{t} A_{t}+L_{r} A_{r}-\nu_{o}\left(x_{3}+x_{2}\right) \geq \delta_{g}>0, \forall t \geq 0 .
$$

Remark 4 Assumptions 1-3 are not restrictive. Since the production choke opening is a control input, Assumption 1 can be satisfied by the construction of the control law. Of course, $\delta_{u}$ has consequences for the solvability of the state feedback regulation problem, and must therefore be sufficiently small. The first condition in (18) is always satisfied in practice, since there is a steady flow 
of gas into the annulus. The second condition in (18) is imposed to deal with the fact that the model of the compressible multiphase flow in the tubing is invalid when the fluid is incompressible, which corresponds to $x_{2}=0$, since the pressure calculation is based on the ideal gas law. From a practical point of view there will always be some gas in the tubing, so this assumption is no restriction. The third condition in (18) states that the reservoir pressure must be high enough for oil to rise above the gas injection point in the tubing. The last assumption, Assumption 3, imposes an upper bound on the gas density in the tubing. For practical gas-lift oil wells, the density of gas will always be less than the density of oil under normal operation.

Lemma 5 ([2, Lemma 2]) Let $x=0$ be an equilibrium point for the nonlinear system

$$
\dot{x}=f(t, x), \quad x\left(t_{0}\right)=x_{0}
$$

where $f:\left[t_{0}, \infty\right) \times \mathbb{R}^{n} \rightarrow \mathbb{R}^{n}$ is piecewise continuous in $t$ and locally Lipschitz in $x$. Let $V:\left[t_{0}, \infty\right) \times \mathbb{R}^{n} \rightarrow \mathbb{R}_{+}$be a continuously differentiable function such that

$$
\begin{aligned}
k_{1}\|x\|^{c} & \leq V(t, x) \leq k_{2}\|x\|^{c} \\
\frac{\partial V}{\partial t}+\frac{\partial V}{\partial x} f(t, x) & \leq-k_{3}\|x\|^{c}+g(\|x\|) \sigma\left(\left\|x\left(t_{0}\right)\right\|, t-t_{0}\right)
\end{aligned}
$$

$\forall t \geq t_{0}, \forall x \in \mathbb{R}^{n}$, where $k_{1}, k_{2}, k_{3}$, and $c$, are strictly positive constants, $g: \mathbb{R}_{+} \rightarrow \mathbb{R}$ is continuous, and $\sigma$ is a class $\mathcal{K} \mathcal{L}$ function satisfying

$$
\int_{t_{0}}^{\infty} \sigma(r, s) d s \leq \sigma_{\infty} r
$$

for some constant $\sigma_{\infty}$. Suppose that there exist constants $k>0$, and $r \geq 0$ such that $k\|x\|^{c} \geq g(\|x\|), \forall\|x\| \geq r$. Then, the equilibrium point $x=0$ of (20) is globally uniformly asymptotically stable. Moreover, solutions of (20) satisfy

$$
\|x(t)\| \leq C e^{-\frac{k_{3}}{c k_{1}}\left(t-t_{0}\right)},
$$

where $C$ depends on the initial state and $\sigma_{\infty}$.

Lemma 6 Solutions of system (1)-(3) are bounded in the sense that there exists a constant $B$, depending on the initial state, such that

$$
x_{i} \leq B\left(x\left(t_{0}\right)\right), i=1,2,3, \forall t \geq 0 .
$$

In particular,

$$
x_{3}<\rho_{o}\left(L_{t} A_{t}+L_{r} A_{r}\right), \forall t \geq 0 .
$$

Proof. It is easily shown that the Lyapunov function candidate $V=2 x_{1}+$ $x_{2}+x_{3}$ is strictly negative for sufficiently large $V$. 
The following theorem states the main result when the flow through the production choke is measured, that is $y_{3}(t)=w_{p c}(t)$.

Theorem 7 Solutions $\hat{x}(t)=\left(\hat{x}_{2}(t), \hat{x}_{3}(t)\right)$ of the observer

$$
\begin{aligned}
\dot{\hat{z}}_{1}= & w_{g c}-\frac{\hat{z}_{1}-y_{1}}{\hat{z}_{2}-y_{1}} y_{3}+k_{1}\left(\hat{z}_{1}, \hat{z}_{2}, y_{1}, y_{2}\right), \\
\dot{z}_{2}= & w_{g c}+C_{r}\left(p_{r}-\rho_{o} g L_{r}+\frac{A_{r}}{A_{t}} \rho_{o} g L_{r}+\frac{g}{A_{t}} y_{1}-y_{2}-\frac{g}{A_{t}} \hat{z}_{2}\right) \\
& \quad-y_{3}+k_{2}\left(\hat{z}_{2}, u, v, y_{1}, y_{2}\right), \\
\hat{z}_{1} \geq & \delta_{2}+y_{1}, \text { and } \hat{z}_{2} \geq \rho_{o} L_{r} A_{r}+\delta_{3}+\hat{z}_{1}, \\
\hat{x}_{2}= & \hat{z}_{1}-y_{1}, \\
\hat{x}_{3}= & \hat{z}_{2}-\hat{z}_{1},
\end{aligned}
$$

where the output injections, $k_{1}$ and $k_{2}$, are given by

$$
\begin{gathered}
k_{1}\left(\hat{z}_{1}, \hat{z}_{2}, y_{1}, y_{2}\right)=c_{1}\left(\frac{M}{R T_{t}}\left(L_{t} A_{t}+L_{r} A_{r}-\nu_{o}\left(\hat{z}_{2}-\hat{z}_{1}\right)\right) y_{2}-\left(\hat{z}_{1}-y_{1}\right)\right) \\
k_{2}\left(\hat{z}_{2}, u, y_{1}, y_{2}, y_{3}\right)=c_{2}\left(\left(\frac{y_{3}}{C_{p c} u}\right)^{2}-\frac{\hat{z}_{2}-y_{1}-\rho_{o} L_{r} A_{r}}{L_{t} A_{t}}\left(y_{2}-p_{s}\right)\right)
\end{gathered}
$$

converge to the actual state $x(t)=\left(x_{2}(t), x_{3}(t)\right)$ exponentially fast in the following sense

$$
\|x(t)-\hat{x}(t)\| \leq C e^{-\gamma\left(t-t_{0}\right)}
$$

where $C$ depends on initial conditions, and

$$
\gamma=\min \left\{c_{1} \frac{\delta_{g}}{L_{t} A_{t}+L_{r} A_{r}}, \frac{C_{r} g}{A_{t}}+c_{2} \frac{\delta_{p}}{L_{t} A_{t}}\right\} .
$$

$\delta_{p} \geq 0$ is a constant satisfying $\max \left\{0, p_{t}-p_{s}\right\} \geq \delta_{p}$ for all $t \geq t_{0}$.

Proof. Define $z_{2}=x_{1}+x_{2}+x_{3}$, which is the total amount of mass in the system. From (1)-(3), (9), (15), and (14), its time derivative is

$$
\dot{z}_{2}=w_{g c}+C_{r}\left(p_{r}-\rho_{o} g L_{r}+\frac{A_{r}}{A_{t}} \rho_{o} g L_{r}+\frac{g}{A_{t}} y_{1}-y_{2}-\frac{g}{A_{t}} z_{2}\right)-y_{3} .
$$

We estimate $z_{2}$ by $\hat{z}_{2}$, which is governed by

$$
\dot{\hat{z}}_{2}=w_{g c}+C_{r}\left(p_{r}-\rho_{o} g L_{r}+\frac{A_{r}}{A_{t}} \rho_{o} g L_{r}+\frac{g}{A_{t}} y_{1}-y_{2}-\frac{g}{A_{t}} \hat{z}_{2}\right)-y_{3}+k_{2}(\cdot),
$$


where $k_{2}(\cdot)$ is an output injection term to be determined. The observer error, $e_{2}=z_{2}-\hat{z}_{2}$, is governed by

$$
\dot{e}_{2}=-\frac{C_{r} g}{A_{t}} e_{2}-k_{2}(\cdot) .
$$

Take the Lyapunov function candidate $V_{2}=\frac{1}{2} e_{2}^{2}$. Its time derivative along solutions of $(38)$ is

$$
\dot{V}_{2}=e_{2}\left(-\frac{C_{r} g}{A_{t}} e_{2}-k_{2}(\cdot)\right)
$$

Selecting

$$
k_{2}\left(\hat{z}_{2}, u, y_{1}, y_{2}, y_{3}\right)=c_{2}\left(\left(\frac{y_{3}}{C_{p c} u}\right)^{2}-\frac{\hat{z}_{2}-y_{1}-\rho_{o} L_{r} A_{r}}{L_{t} A_{t}} \max \left\{0, y_{2}-p_{s}\right\}\right)
$$

where $c_{2}>0$, and inserting (40) into (39), we get

$$
\dot{V}_{2}=-\left(\frac{C_{r} g}{A_{t}}+c_{2} \frac{\max \left\{0, y_{2}-p_{s}\right\}}{L_{t} A_{t}}\right) e_{2}^{2}
$$

So we obtain

$$
\left\|e_{2}(t)\right\| \leq\left\|e_{2}\left(t_{0}\right)\right\| e^{-\frac{C_{r} g}{A_{t}}\left(t-t_{0}\right)} .
$$

Next, define $z_{1}=x_{1}+x_{2}$, which is the total mass of gas in the system. From (1) $-(2)$, its time derivative is

$$
\dot{z}_{1}=w_{g c}-\frac{z_{1}-y_{1}}{z_{2}-y_{1}} y_{3}
$$

We estimate $z_{1}$ by $\hat{z}_{1}$, which is governed by

$$
\dot{\hat{z}}_{1}=w_{g c}-\frac{\hat{z}_{1}-y_{1}}{\hat{z}_{2}-y_{1}} y_{3}+k_{1}(\cdot),
$$

where $k_{1}(\cdot)$ is an output injection term to be determined. The observer error, $e_{1}=z_{1}-\hat{z}_{1}$, is governed by

$$
\dot{e}_{1}=-\frac{z_{1}-y_{1}}{z_{2}-y_{1}} y_{3}+\frac{\hat{z}_{1}-y_{1}}{\hat{z}_{2}-y_{1}} y_{3}-k_{1}(\cdot) .
$$

Notice that the observer error dynamics (38) and (45), is in a cascaded form, where the dynamics of $e_{2}$ is independent of $e_{1}$. Since $e_{2}$ converges to zero, we will seek to apply Lemma 5 . Towards that end, we take the Lyapunov function candidate $V_{1}=\frac{1}{2} e_{1}^{2}$. Its time derivative along solutions of (45) is

$$
\dot{V}_{1}=-\frac{y_{3}}{z_{2}-y_{1}} e_{1}^{2}+y_{3} \frac{\hat{z}_{1}-y_{1}}{\left(z_{2}-y_{1}\right)\left(\hat{z}_{2}-y_{1}\right)} e_{1} e_{2}-e_{1} k_{1}(\cdot)
$$


We now select

$$
k_{1}\left(\hat{z}_{1}, \hat{z}_{2}, y_{1}, y_{2}\right)=c_{1}\left(\frac{M}{R T_{t}}\left(L_{t} A_{t}+L_{r} A_{r}-\nu_{o}\left(\hat{z}_{2}-\hat{z}_{1}\right)\right) y_{2}-\hat{z}_{1}+y_{1}\right),
$$

where $c_{1}>0$, and obtain

$$
\begin{aligned}
\dot{V}_{1}= & -\left(\frac{y_{3}}{z_{2}-y_{1}}+c_{1} \frac{L_{t} A_{t}+L_{r} A_{r}-\nu_{o}\left(z_{2}-y_{1}\right)}{L_{t} A_{t}+L_{r} A_{r}-\nu_{o}\left(z_{2}-z_{1}\right)}\right) e_{1}^{2} \\
& +\left(y_{3} \frac{\hat{z}_{1}-y_{1}}{\left(z_{2}-y_{1}\right)\left(\hat{z}_{2}-y_{1}\right)}-c_{1} \frac{\nu_{o}\left(z_{1}-y_{1}\right)}{L_{t} A_{t}+L_{r} A_{r}-\nu_{o}\left(z_{2}-z_{1}\right)}\right) e_{1} e_{2} .
\end{aligned}
$$

Using Lemma 6, Assumptions 2 and 3, and noticing that $\left(\hat{z}_{1}-y_{1}\right) /\left(\hat{z}_{2}-y_{1}\right)<$ 1, we obtain

$$
\dot{V}_{1} \leq-\left(\frac{y_{3}}{2 B}+c_{1} \frac{\delta_{g}}{L_{t} A_{t}+L_{r} A_{r}}\right) e_{1}^{2}+\left(\frac{y_{3}}{\delta_{2}+\delta_{3}}+c_{1} \frac{B \nu_{o}}{\delta_{g}}\right)\left\|e_{1}\right\|\left\|e_{2}\right\| .
$$

We can now apply Lemma 5 with $V=\left(e_{1}^{2}+e_{2}^{2}\right) / 2$,

$$
\begin{aligned}
k_{3} & =\min \left\{c_{1} \frac{\delta_{g}}{L_{t} A_{t}+L_{r} A_{r}}, \frac{C_{r} g}{A_{t}}+c_{2} \frac{\delta_{p}}{L_{t} A_{t}}\right\}, \\
g(\|e\|) & =\left(\frac{y_{3}}{\delta_{2}+\delta_{3}}+c_{1} \frac{B \nu_{o}}{\delta_{g}}\right)\left\|e_{1}\right\|, \\
\sigma\left(\left\|e\left(t_{0}\right)\right\|, t-t_{0}\right) & =\left\|e_{2}\left(t_{0}\right)\right\| e^{-\frac{C_{r} g}{A_{t}}\left(t-t_{0}\right)}
\end{aligned}
$$

to achieve the desired result, and in particular the estimate (35).

In the case when the density is measured, that is $y_{3}(t)=\rho_{m}(t)$, we can simply replace $y_{3}$ with

in $(27)-(28)$ and $(33)$.

$$
C_{p c} \sqrt{y_{3} \max \left\{0, y_{2}-p_{s}\right\}} u
$$

A key feature of the observer design is that it is independent of the flow of gas from the annulus to the tubing. This is important, because it provides robustness with respect to modelling errors of this internal flow. It is due to the coordinate change $x_{1}+x_{2} \rightarrow z_{1}, x_{1}+x_{2}+x_{3} \rightarrow z_{2}$, and the fact that the dynamics of $\left(z_{1}, z_{2}\right)$ is independent of $(5)$.

\subsection{Open-loop Simulations}

The numerical coefficients used in the simulations of system (1)-(3) are taken from a full-scale gas-lift well of depth approximately 2 kilometers. For this 
a)

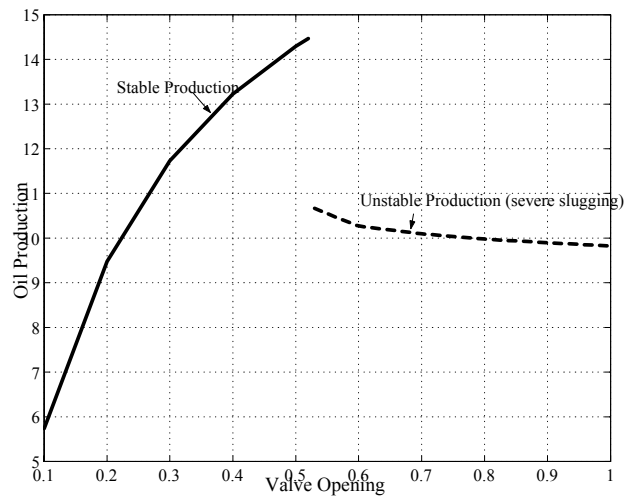

b)

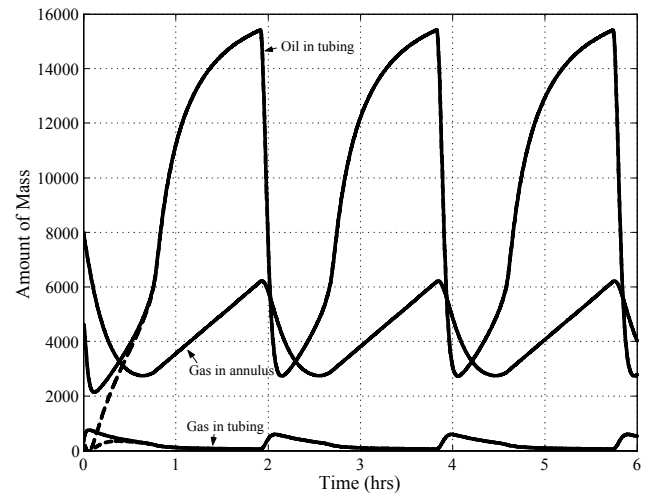

Fig. 3. a) Mean oil production versus production choke opening. Solid line indicates stable production and dashed line indicates severe slugging. b) States (solid line) and their estimates (dashed line) for the system during severe slugging.

case, simulations have been performed to calculate mean oil production as a function of production choke opening. The result is presented in Figure 3a. The production is stable for small choke openings and increases as the choke opening is increased. At a choke opening of about 0.52 , the flow becomes unstable and goes into severe slugging, leading to a dramatic loss of production. The increasing trend of the production for small choke openings, suggests that a higher production is possible for large choke openings if the flow can be stabilized. This is shown to be the case in the next section. In this section, we will illustrate the performance of the observer by running open-loop simulations for the nominal case of perfect model. Figure 3b shows the states along with the estimates for the tubing over a six hour simulation with $c_{1}=0$ and $c_{2}=0$. The flow is clearly in the state of severe slugging, and the estimates converge to the actual states. The first row of graphs in Figure 4 shows the details over the first hour for this case. Estimates are good within 0.7 hours. In the second row of graphs in Figure $4, c_{1}=0$ and $c_{2}=0.001$, and convergence is much faster. Estimates are good within 8 minutes. Setting $c_{1}=1.43$ (keeping $\left.c_{2}=0.001\right)$ increases the convergence rate further, as the third row of graphs in Figure 4 shows. Estimates are in this case good within 3 seconds. It is clear that the convergence rate estimate $\gamma$, as defined in (35) is very conservative, since it is equal to 0 in the first two cases $\left(c_{1}=0\right)$. However, looking at inequality (49), the flow through the production choke, $v$, defines a better bound for the estimation convergence rate, and explains why the observer converges with $c_{1}=0$. Although $\gamma$ is a very conservative estimate for the convergence rate, (35) tells us that our observer can achieve any desired convergence rate by increasing $c_{1}$ and $c_{2}$. From the proof of Theorem 7 , we see that $c_{2}$ governs the convergence rate of the estimate for the total mass in the system (gas and oil), whereas $c_{1}$ governs the convergence rate of the estimate for the total mass of gas in the system, but with an upper bound governed by $c_{2}$. 
$c_{1}=0$ and $c_{2}=0$
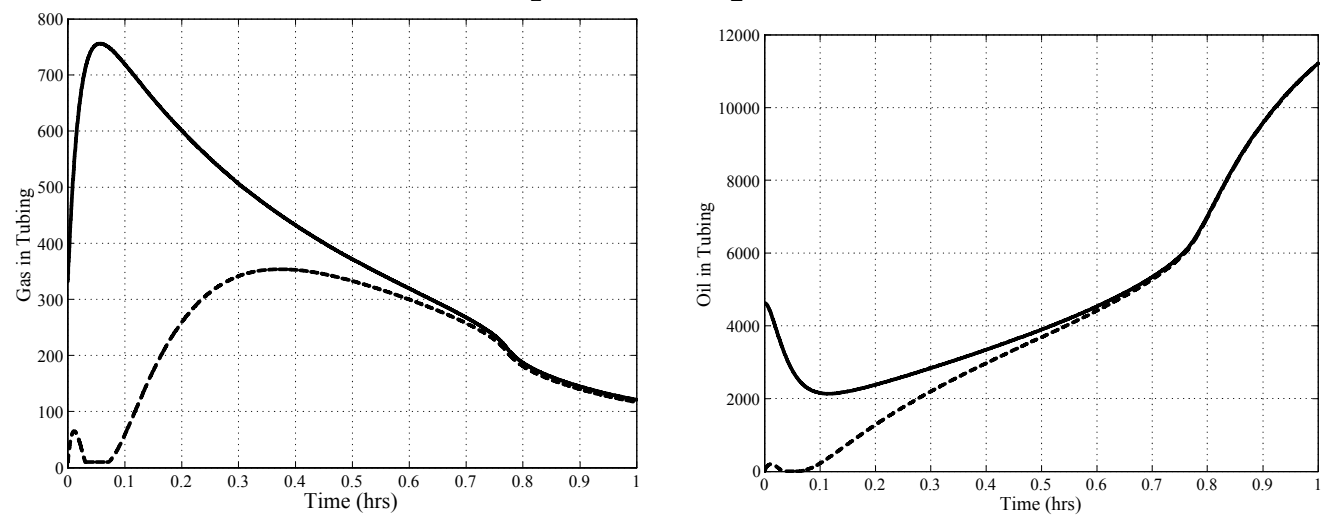

$c_{1}=0$ and $c_{2}=0.001$
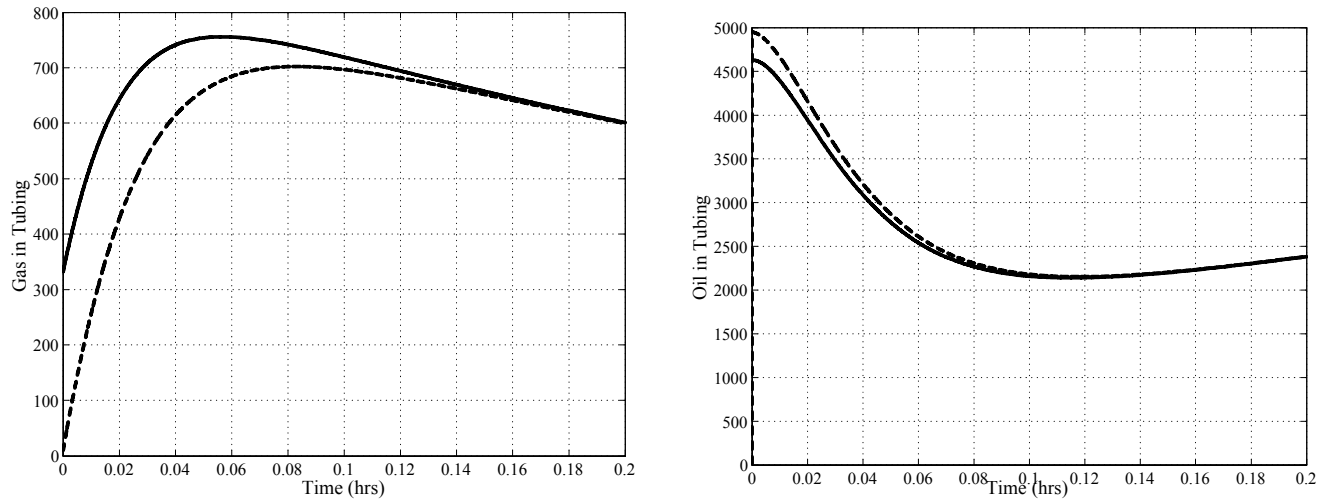

$c_{1}=1.43$ and $c_{2}=0.001$
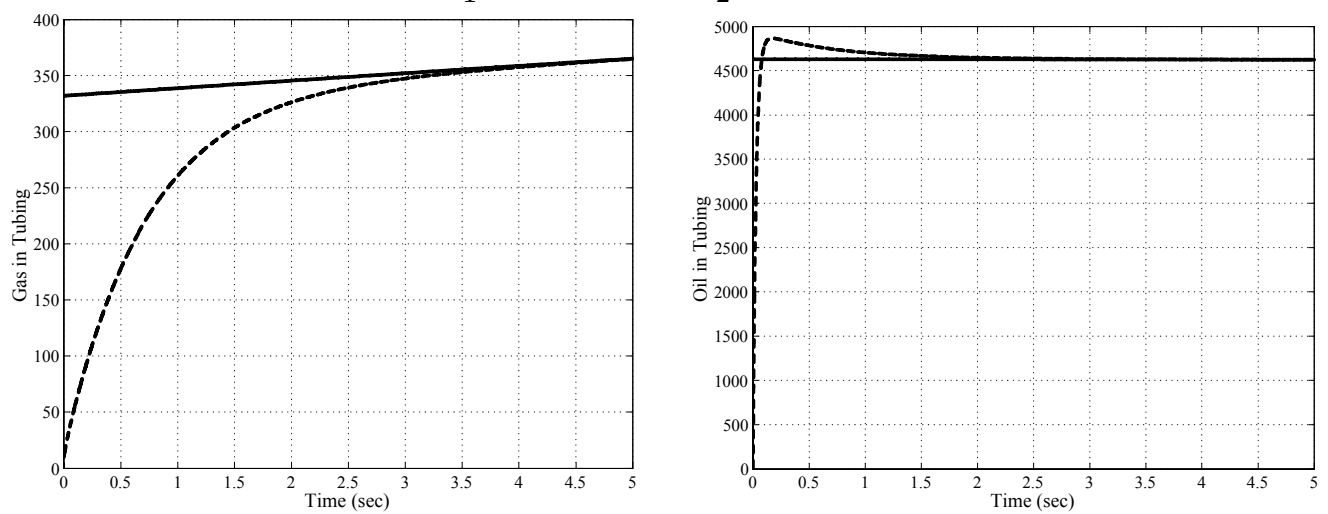

Fig. 4. Details of estimates for various choices of $c_{1}$ and $c_{2}$. Notice the different time scales in the three cases.

\section{Anti-slug Control by Output Feedback}

It has been shown in [3] that severe slugging can be attenuated by stabilizing the downhole pressure using a control law of the form

$$
u=u^{*}+K\left(p_{t, b}-p_{t, b}^{*}\right)
$$


a)

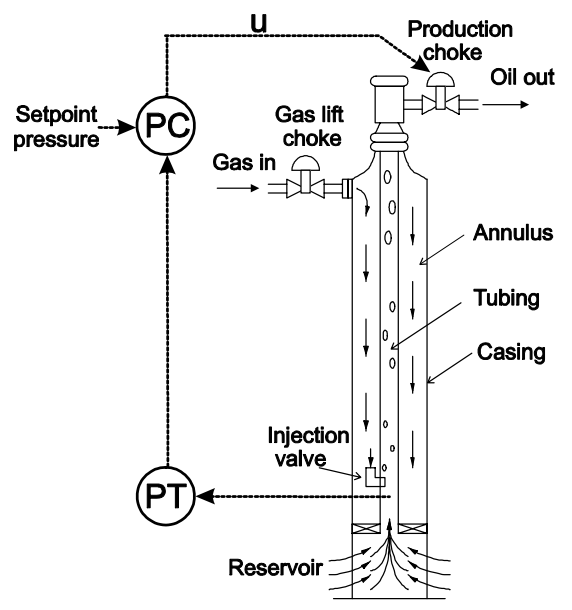

b)

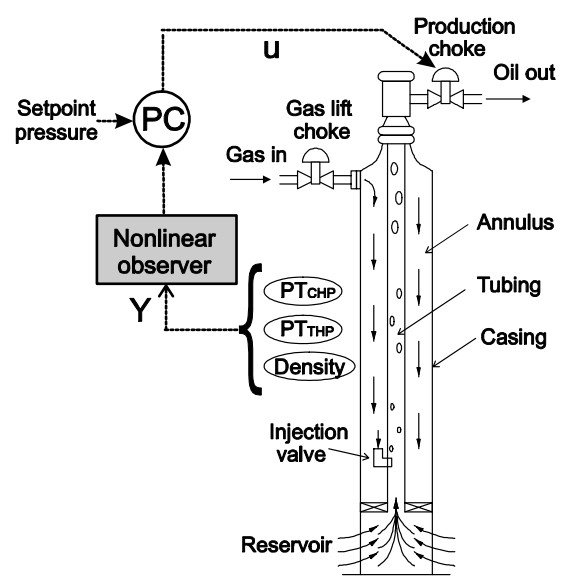

Fig. 5. Conventional control structure with downhole pressure measurement (a), and observer-based control structure with top-side information, only (b).

where $u^{*}$ and $p_{t, b}^{*}$ are some appropriate constants ${ }^{2}$. This control configuration is sketched in Figure 5a. The downhole pressure is in general not available as a measurement, and neither are the individual states in the tubing. However, we may replace the states in (15) by their estimates generated by the observer designed in the previous section, to obtain an estimate of the downhole pressure. The controller obtained by using this estimate in place of $p_{t, b}$ in (54), is called the certainty equivalence controller. For linear systems, stability of the closed loop using the certainty equivalence controller is guaranteed by the separation principle of linear systems. For general nonlinear systems, however, not even an exponentially convergent observer in conjunction with an exponentially stabilizing state feedback control law can guarantee stability of the closed loop system. Stability of the closed loop system obtained by combining our observer with some state feedback control law must therefore be checked in each specific case. Since the topic of this paper is observer design, we will not persue a mathematically rigorous proof of stability for the closed loop system using our observer in conjunction with (54). We will point out, however, that (54) becomes practically implementable by means of our observer, and that the resulting controller successfully stabilizes the closed loop system in laboratory experiments, results from which are presented next.

$\overline{2}$ Usually, $p_{t, b}^{*}$ is the setpoint chosen by the operator, while $u^{*}$ is the resulting steady state choke opening adapted to by adding slow integral action to (54). 


\section{$5 \quad$ Laboratory Experiments}

Realistic tests of control structures for gas-lift wells are performed using the gas-lift well laboratory setup at TU Delft ${ }^{3}$. Results from prior experiments show that stabilization of the multiphase flow in the tubing can be achieved by a conventional PI controller adjusting the production choke opening based on measurement of the downhole pressure. The control structure applied in the laboratory experiments controls the estimated downhole pressure, given by the observer, and manipulates the opening of the production choke. The control structure is given in Figure $5 \mathrm{~b}$.

\subsection{Experimental Setup}

The laboratory installation represents a gas-lift well, using compressed air as lift gas and water as produced fluid. The production tube is transparent, facilitating visual inspection of the flow phenomena occurring as control is applied. The production tube measures $18 \mathrm{~m}$ in height and has an inner diameter of $20 \mathrm{~mm}$. The fluid reservoir is represented by a tube of the same height and an inner diameter of $80 \mathrm{~mm}$. The reservoir pressure is given by the static height of the fluid in the reservoir tube. A 30 litre gas bottle represents the annulus, with the gas injection point located at the bottom of the production tube. In the experiments run in this study, gas is fed into the annulus at a constant rate of $11 \mathrm{~L} / \mathrm{min}$ (under standard conditions: $25^{\circ} \mathrm{C}, 1$ bara). Input and output signals to and from the installation are handled by a microcomputer system, to which a laptop computer is interfaced for running the control algorithm and presenting output.

\subsection{PI Controller and Observer}

In the laboratory, density in the top of the tubing, $\rho_{m}(t)$, is available as a measurement ${ }^{4}$. Therefore, the observer given in Theorem 7 , with $y_{3}$ replaced by (53), was used for estimation of downhole pressure. The estimated downhole pressure of the gas-lift well was stabilized using the digital PI controller

3 The experimental setup is designed and implemented by Shell International Exploration and Production B.V., Rijswijk, and is now located in the Kramers Laboratorium voor Fysische Technologie, Faculty of Applied Sciences, Delft University of Technology.

4 The density measurement is derived from two pressure measurements in the upper half of the tubing, and is therefore an approximation to the actual density in the top of the tubing. 


$$
\Delta u_{k}=K_{c}\left[\left(e_{k}-e_{k-1}\right)+\frac{\Delta t}{\tau_{I}} e_{k}\right] .
$$

The gains and integral times applied by the controller are given in Table 1, and the observer gains are given in Table 2. The PI-controller has varying gain. Note, however, that hysteresis is implemented to prevent frequent gain changes due to noise in the pressure estimate.

Table 1

Gain scheduling

\begin{tabular}{|c|c|c|}
\hline Valve Opening & Gain & Integral Time \\
\hline $55 \% \leq u<65 \%$ & -0.5 & $150 \mathrm{sec}$ \\
\hline $65 \% \leq u<73 \%$ & -1.5 & $150 \mathrm{sec}$ \\
\hline $73 \% \leq u<100 \%$ & -2.0 & $200 \mathrm{sec}$ \\
\hline
\end{tabular}

Table 2

Gains - Observer

\begin{tabular}{|c|c|}
\hline Total mass of gas, $c_{1}$ & Total mass, $c_{2}$ \\
\hline 0.5 & 0.01 \\
\hline
\end{tabular}

\subsection{Experimental Results}

The laboratory experiment followed the control sequence given in Table 3. The multiphase flow is initially open-loop stabilized by applying a small choke opening, causing the pressure drop to be friction dominated. When steadystate is reached the controller is turned on. It gradually increases the choke opening and moves the system into the open-loop unstable domain in order to reach the pressure setpoint. When steady-state is reached again, this time at a large choke opening, the controller is turned off, leaving the choke with the last controlled opening. This is why oscillations appear at the end of the time series below, confirming that the new operating point is indeed in the open-loop unstable domain.

Table 3

The control sequence

\begin{tabular}{|c|c|c|}
\hline Time Slot & Control & Valve Opening \\
\hline$(-5) \mathrm{min}-0 \mathrm{~min}$ & open loop & $55 \%$ \\
\hline $0 \mathrm{~min}-50 \mathrm{~min}$ & closed loop & controlled \\
\hline $50 \mathrm{~min}-55 \mathrm{~min}$ & open loop & $83.2 \%$ \\
\hline
\end{tabular}


a)

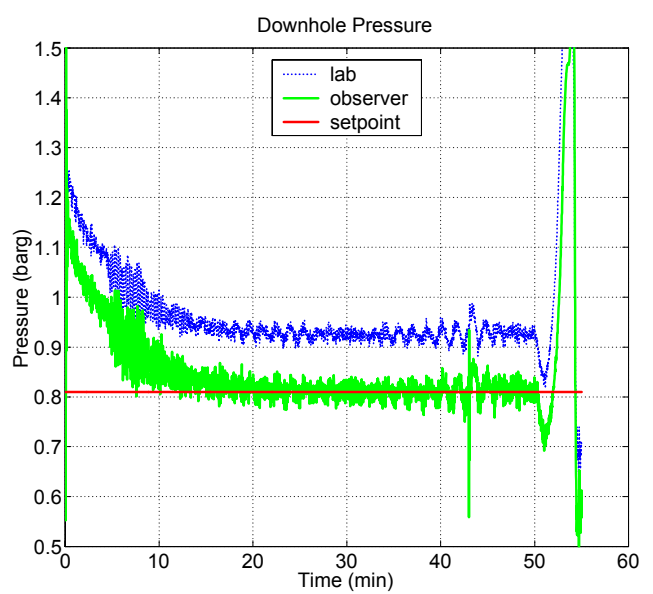

b)

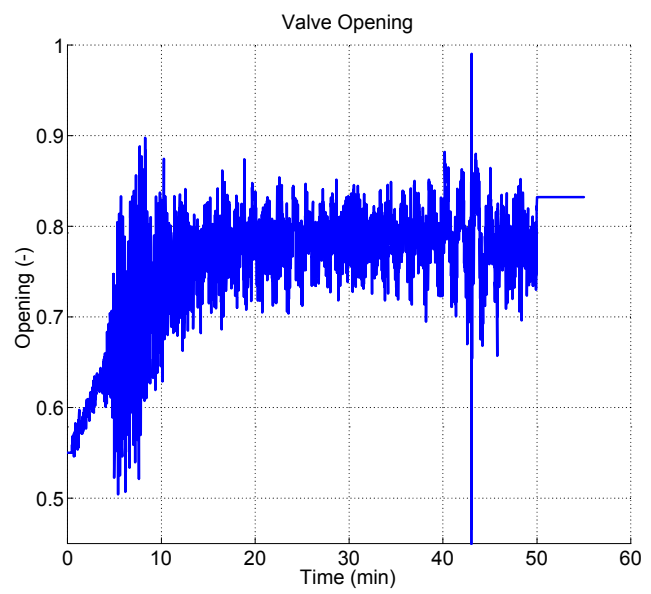

Fig. 6. a) Estimated downhole pressure given by the observer and measured downhole pressure. b) Opening of the production choke.

The estimated downhole pressure from the observer and the measured downhole pressure are shown Figure 6a. The results show that the estimated downhole pressure is stabilized, with the steady-state production choke opening at $79 \%$ (see Figure 6b). The valve opening fluctuates somewhat due to noise in the downhole pressure estimate. After 43 minutes a large external disturbance to the gas supply source of the system is introduced, as can be seen from the peaks in the experimental results. The controller successfully attenuates the disturbance, indicating robustness.

The measurements used by the observer, that is the casing head pressure, the tubing head pressure, and the fluid density, are shown in Figures 7-8a. The measurements of the tubing head pressure and the density are low-pass filtered, using a cut-off period of $T_{c, T H P}=20$ seconds and $T_{c, \rho}=10$ seconds, respectively.

The flow rate from the well is shown in Figure 8b, and corresponds to an average production choke opening of $79 \%$. The average open-loop liquid production as a function of production choke opening has been produced by a series of experiments and is shown in Figure 9. For valve openings less than about $70 \%$, the production is open-loop stable, while for openings larger than $70 \%$, the production is open-loop unstable, and is significantly lower compared to the production at $70 \%$ valve opening. It is interesting to notice that the production curve from the gas-lift laboratory shows the same qualitative behaviour as the one predicted by system (1)-(3) for a full-scale gas-lift oil well (recall Figure 3a). The cross at $79 \%$ choke opening in Figure 9 gives the production for the controlled experiment. The key information in the figure, and the main result of the experimental part of this paper, is in comparing the production for the controlled experiment with the maximum achievable 
a)

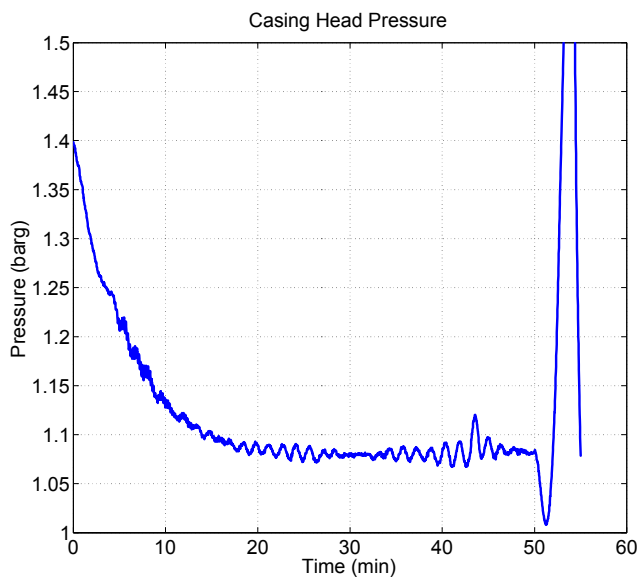

b)

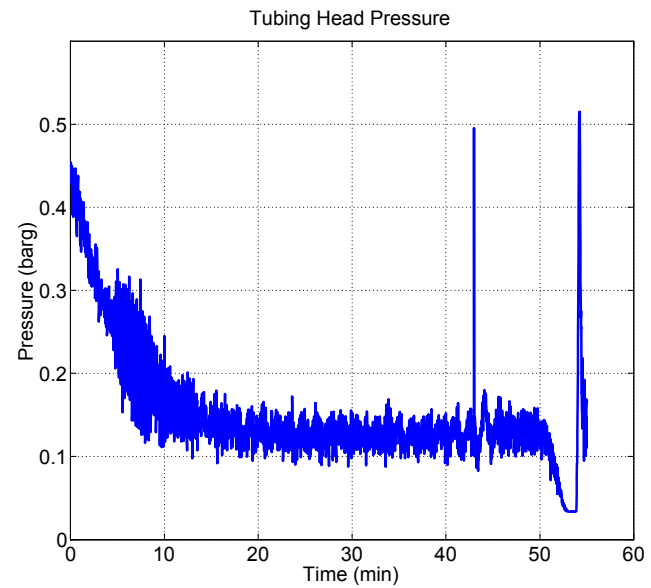

Fig. 7. a) Pressure in the annulus. b) Pressure in the top of the tubing.

a)

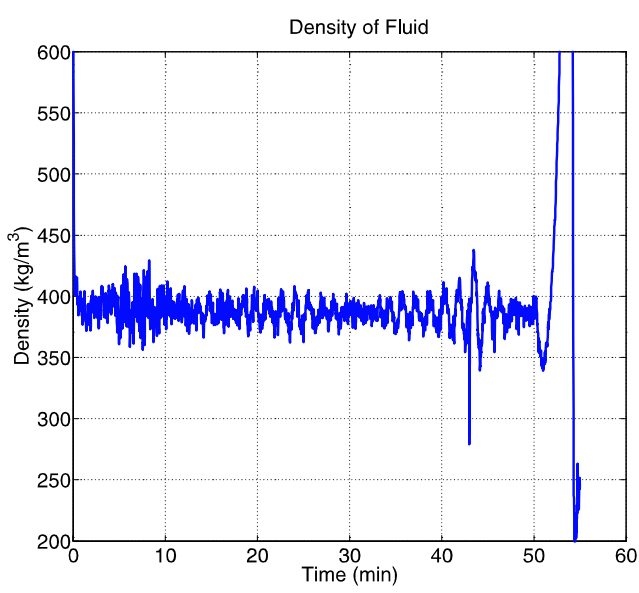

b)

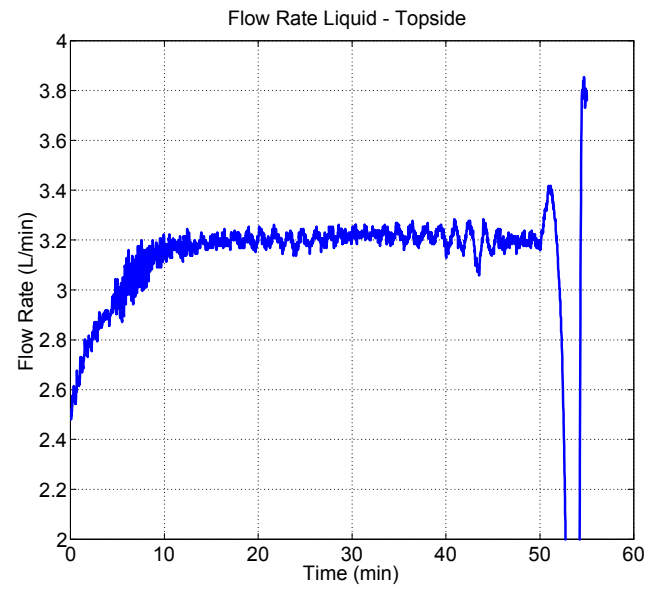

Fig. 8. a )Fluid density in the top of the tubing. b) Total fluid production.

open-loop production obtained for $70 \%$ choke opening: The increase in produced fluid gained by stabilizing the gas-lift well at $79 \%$, by feedback control, is about $5 \%$.

\section{Conclusions}

In this paper, we have designed and analyzed a reduced order nonlinear observer for the states of the multiphase flow in the tubing. The observer relies on topside measurements, only. A key feature of the design is that it exploits the structure of the model to obtain robustness with respect to the internal flow between the annulus and the tubing. The performance of the observer was demonstrated in simulations. The practical applicability of a control scheme 


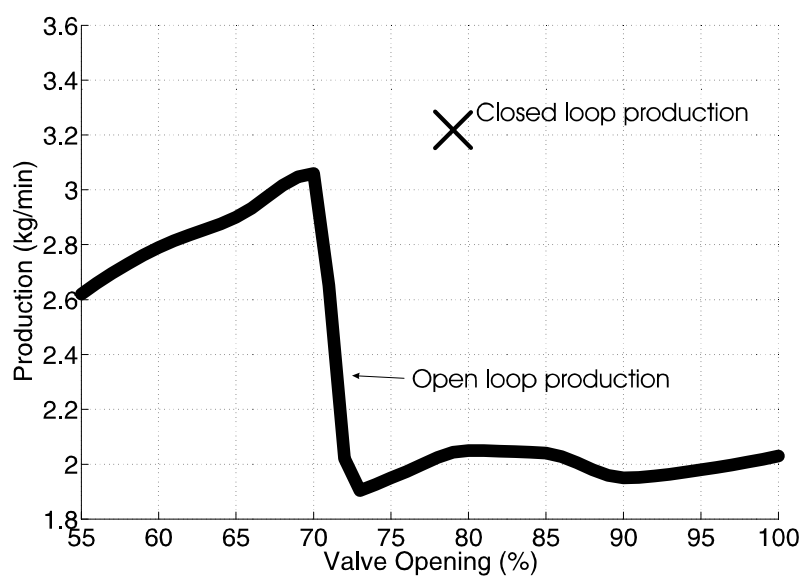

Fig. 9. Production achieved from the gas-lift laboratory by closed loop compared to open loop production.

consisting of our observer in conjunction with conventional control techniques was demonstrated in laboratory experiments, where the multiphase flow in the tubing was successfully stabilized using top-side information, only. The resulting production rate was $5 \%$ higher than the open-loop maximum production rate.

The results of this paper clearly show that there is a potential for increasing production from gas-lift oil wells by installing a relatively simple control system.

\section{Acknowledgements}

We gratefully acknowledge the support from Shell International Exploration and Production B.V., and Kramers Laboratorium voor Fysische Technologie, Faculty of Applied Sciences, Delft University of Technology. In particular, we would like to thank Dr. Richard Fernandes (Shell) and Prof. Dr. R.V.A. Oliemans (TU Delft). Also, we thank Dr. Lars Imsland (NTNU) for valuable discussions.

\section{References}

[1] M. Dalsmo, E. Halvorsen, and O. Slupphaug, "Active feedback control of unstable wells at the Brage field," SPE paper no. 77650.

[2] O.M. Aamo, M. Arcak, T.I. Fossen, and P.V. Kokotovic, "Global output tracking control of a class of Euler-Lagrange systems with monotonic nonlinearities in the velocities," International Journal of Control, vol. 74, no. 7, 
pp. 649-658, 2001.

[3] G.O. Eikrem, B. Foss, L. Imsland, B. Hu, and M. Golan, "Stabilization of gas lifted wells," Proceedings of the 15th IFAC World Congress, Barcelona, Spain, 2002 .

[4] G.O. Eikrem, L. Imsland, and B. Foss, "Stabilization of gas lifted wells based on state estimation," Proceedings of the International Symposium on Advanced Control of Chemical Processes, Hong Kong, China, 2004.

[5] K. Havre, K.O. Stornes, and H. Stray, "Taming slug flow in pipelines," $A B B$ Review, no. 4, 2000.

[6] K. Havre and M. Dalsmo, "Active feedback control as the solution to severe slugging," SPE paper no. 71540.

[7] L. Imsland, Topics in Nonlinear Control: Output Feedback Stabilization and Control of Positive Systems, Ph.D. thesis, NTNU, 2002.

[8] F.E. Jansen, O. Shoham, and Y. Taitel, "The elimination of severe slugging Experiments and modeling," International Journal of Multiphase Flow, vol. 22, no. 6, pp. 1055-1072, 1996.

[9] B. Jansen, M. Dalsmo, L. Nøkleberg, K. Havre, V. Kristiansen, and P. Lemetayer, "Automatic control of unstable gas lifted wells," SPE paper no. 56832 .

[10] W.J.G.J. Kinderen and C.L. Dunham, "Real-time artificial lift optimization," SPE paper no. 49463.

[11] S.I. Sagatun, "Riser slugging - a mathematical model and the practical consequences," submitted to SPE.

[12] C. Sarica and J.Ø. Tengesdal, "A new technique to eliminate severe slugging in pipeline/riser systems," SPE paper no. 63185.

[13] G. Skofteland and J.-M. Godhavn, "Suppression of slugs in multiphase flow lines by active use of topside choke - Field experience and experimental results," Proceedings of Multiphase'03, San Remo, Italy, 2003.

[14] H.I. Berge and G. Halvorsen, "Downhole monitoring \& acquisition," presentation at NTNU on January 29, 2004, http://www.imn.no/filer/1075472044-Statoils_situasjon_presentasjon_ifbm_idekonk.pdf (in Norwegian).

[15] E. Storkaas, S. Skogestad, and V. Alstad, "Stabilizing of desired flow regimes in pipelines," Proceedings of AIChE Annual Meeting, Reno, Nevada, USA, 2001.

[16] Z.G. Xu and M. Golan, "Criteria for operation stability of gas lift," SPE paper no. 19362. 